\title{
Reducing Packet Losses in Mobile Ad Hoc Network Using the Warning Message Generated from a Routing Node
}

\author{
Rezvi Shahariar ${ }^{* 1}$ and Abu Naser ${ }^{2}$ \\ ${ }^{1}$ Institute of Information Technology, Dhaka University, Dhaka 1000, Bangladesh \\ ${ }^{2}$ Department of Computer Science and Engineering, Shahjalal University of Science \& Technology, Sylhet, Bangladesh
}

(Received: 22 September 2013; Accepted: 6 April 2014)

\begin{abstract}
In mobile ad hoc network communication is performed usually by using only send and receive messages and every node is powered by limited energy from low capacity battery. Every send or receive message takes particular amount of energy from the node. So node's total energy level gradually decreases each time while it is sending or receiving something. In this way node will die out and packets coming from the source will be dropped since one of the routing node on the current route is no longer functioning. These packet loss events are observed and minimized in this paper. In the proposed approach, when source receives Warning Message from any routing node on the ongoing route then it will stop sending packets on the ongoing route. Critical energy level of routing node has been defined to generate a Warning Message when routing node's energy level reduces to critical energy level.
\end{abstract}

Keywords: Mobile Ad Hoc Network, Routing Protocol, Critical Energy Level, Warning Message

\section{Introduction}

A mobile ad hoc network is a wireless network consisting of mobile nodes and it is managed without any central administration and it can be deployed easily in anywhere. Within a transmission range each node can communicate directly with others. But out of transmission range a node cannot communicate directly. For long distance communication, source uses multiple intermediate nodes to forward packets to destination. Thus routing is a crucial issue to select route from source to destination in a mobile ad hoc network.

Conventional routing protocols used in wired network such as distance vector ${ }^{1}$ or link state ${ }^{2}$ routing protocols require huge periodic broadcast messages to be exchanged between the neighbors. But nodes in a MANET have mobility, low processing/computation power, limited battery energy and also high error rates. Hence new routing protocols are developed for wireless ad hoc networks such as dynamic source routing (DSR) ${ }^{3}$, extended dynamic source routing $(\text { EDSR })^{4}$, ad hoc on demand distance vector $(A O D V)^{5}$, destination-sequenced distance-vector routing (DSDV) ${ }^{6}$, and zone routing protocol (ZRP) ${ }^{7}$ etc.

The rest of the paper is organized as follows: section II reviews related work, section III focuses on the motivation of this research, in section IV proposal is described, section $\mathrm{V}$ illustrates the performance comparison and finally conclusion is drawn in section VI.

\section{Background}

Dynamic source routing (DSR) ${ }^{3}$ is a simple and efficient routing protocol designed specifically for use in multi-hop wireless ad hoc network. The protocol is composed of the two basic procedures which are as follows:

\section{i. $\quad$ Route Discovery \\ ii. Route Maintenance.}

In $\mathrm{DSR}^{3}$ a node wants to send packets to a destination node, it performs route discovery procedure of this protocol to find all the available routes to the destination. When there is an occurrence of any route error, route maintenance procedure is used to provide another route or to rediscover the routes if there is no route in cache of source node. The steps are used in $\mathrm{DSR}^{3}$ is given in the following:

1. Node wants to communicate with a particular destination generates a ROUTE REQUEST message to find the available routes to the destination. The ROUTE REQUEST message appends all the nodes information in it as it is propagated through the network. Finally ROUTE REQUEST message will reach a node which is either the destination node or it will know the destination node.

2. In both cases a ROUTE REPLY message is generated to the originator of ROUTE REQUEST message.

3. From these routes, source node uses only one route to communicate with the destination node.

4. This step will be used if there is an occurrence of route error in the network. When there is an occurrence of a route breakage on the ongoing route is found, the node that detects the route error it will send a ROUTE $E R R O R$ message to the source node. Source node uses another available route immediately from the cache or it will rediscover routes to destination if no route is available in cache of a source node.

In Figure 1, node S will perform Route Discovery procedure of DSR to find available routes between S and D. From these available routes three different routes are shown in Figure 2. At a time only one route will be used by node S. If the current route is broken then another one will be used by node S from its cache. Otherwise another Route Discovery will be performed by S. This is the way how DSR routing protocol actually works. 


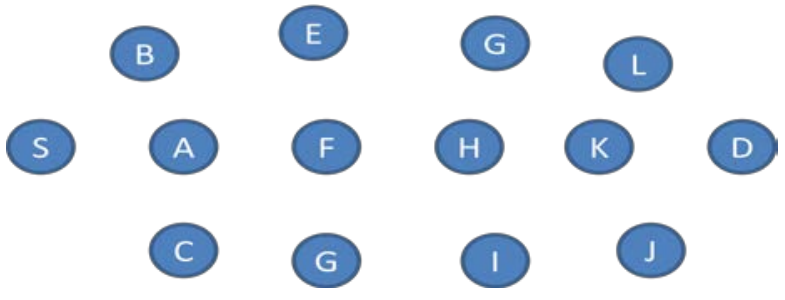

Fig. 1. A MANET environment

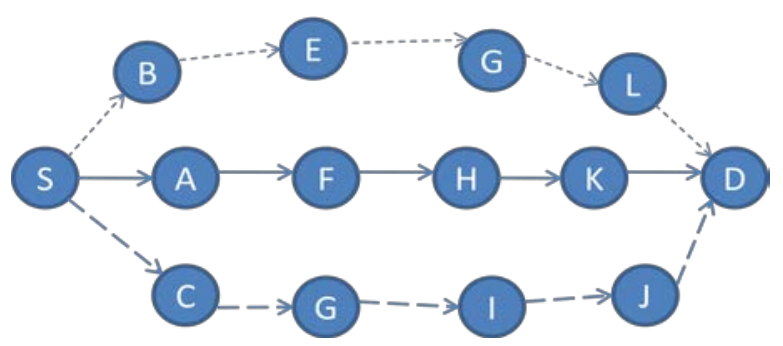

Fig. 2. Three different routes

$\mathrm{EDSR}^{4}$ finds two disjoint paths between the source and destination during route discovery process without producing extra overhead. One route is black and another route is white. A white or black node is a marked node; otherwise it is unmarked node. Initially all nodes except source node are unmarked. Each host maintains a list of tuples (source, destination, request-id, color), where requestid is a sequence number maintained at the sender. Each intermediate host can be either white or black but not both. Destination can be both white and black. Source is initially marked both white and black. $\mathrm{EDSR}^{4}$ is given as an approach in the following:

1. S initially sends two requests (s, d, id, black), (s, d, id, white). When an intermediate node receives (s, d, id, color) it process as the step 2.

2. If $\mathrm{v}$ has been marked for (s, d, id), it will be discarded.

3. Else If $\mathrm{v}$ is unmarked and it receives for the first time and it waits for $t$ unit time.

i. If $\mathrm{v}$ does not receive (s, $\mathrm{d}$, id, !color) within t unit time, $\mathrm{v}$ is marked with color.

ii. If $\mathrm{v}$ receives ( $\mathrm{s}, \mathrm{d}$, id, !color) within $\mathrm{t}$ unit time, $\mathrm{v}$ is marked with random color .

4. Once $v$ is marked it performs one of the followings:

i. If $\mathrm{v}$ is a destination or a route with a matching color from $\mathrm{v}$ to $\mathrm{d}$ exits, $\mathrm{v}$ sends a route reply to $\mathrm{s}$.

ii. Else v appends v's own address to the record and will forward with the committed color.

The destination keeps the first black and first white route and then a route reply is sent from the destination to source.

\section{Motivation}

In DSR and EDSR source node continuously sends packets on the ongoing route. Even if there is a route breakage on the current route source will send packets. Source will not be informed immediately whenever a route is broken. In the time interval between the route breakage occurrence and the receiving of ROUTE ERROR message by the source node, source also sends packets on the ongoing route. All the packets sent in this interval will be dropped from the network since a node will not find the next node on the ongoing route. To reduce these packets losses we define the critical battery energy level of a host. When node's energy level reaches into critical energy level then it will follow some tasks to prevent packets losses. These tasks are given as an approach in the next section.

\section{Reducing Packet Losses in MANETs}

In MANET node is communicating by using sending and receiving of packets. Each time a node sends or receives something it actually uses some energy from battery. In such way a node's battery energy will be finished and this node will die out soon. As a result one of the routing nodes will not find it to forward packets. So, route breakage will occur obviously and some packets will be lost because source will not know the off condition of this node and source node will continue sending packets using this broken route in $\mathrm{DSR}^{3}$ and in $\mathrm{EDSR}^{4}$. This is a scenario of energy abuses and packets losses in MANETs. To get rid from this situation this paper gives an approach to save energy and at the same time reducing packet losses in MANETs.

\section{A. Proposed approach}

A routing node will generate a Warning Message while its energy level reaches to a critical value and tries to forward all the packets to the next node on the same route using its remaining energy. Thus when a routing node's energy level reached to critical level it will send a Warning Message to the source node immediately and relays the received message on the next hop. This low energy node tries to relays all the packets to the next hop on the ongoing route by using its current energy level until its energy reduces to zero level. When the source node receives a Warning Message from a routing node it assumes that the routing node will not be functional long. In this way the source node gets information about the stopping condition of this routing node in our approach. Whenever a node will go in off mode there must be a route breakage event on the ongoing route and also there will be some packets which do not get a way to the destination. These packets losses can be minimized by not sending more packets on the current route because the source node gets information earlier from the low energy node using the Warning Message. The Warning Message is generated by a node when $10 \%$ of total energy remains. On the other hand ROUTE ERROR message is generated when an error detecting node does not find the error node because its energy level is totally empty. Thus we can easily realize that Warning Message comes earlier than ROUTE ERROR message and source can stop sending packets on the current route and this way packet loss can be minimized more by using this approach than $\mathrm{DSR}^{3}$ and its extension ${ }^{4}$.

\section{B. Critical Energy Level and Warning Message}

This paper is introducing critical energy level of a routing node. A node's critical energy level is set based on the following equation: 
$\mathrm{C}_{\mathrm{E}}=0.1 * \mathrm{~T}_{\mathrm{E}}$, Where $\mathrm{C}_{\mathrm{E}}$ denotes critical energy level and $\mathrm{T}_{\mathrm{E}}$ denotes total energy level of a host.

When a node's energy level falls into critical energy level (10\% of its total energy) it will generate a Warning Message to the source node immediately and tries to forward all the packets to the next node on the ongoing route by using its remaining energy. A Warning Message contains information about the low energy node, time to live, and the route information to tell the source that the source node cannot communicate more to the destination by using the energy low node. Hence it will be the responsibility of source node that it will not forward a single packet after receiving a Warning Message from any routing node. Critical energy level of every node is set such that a node can send at least a Warning Message to the source node and tries to forward all the forwarded packets to the next hop by using the remaining energy level.

C. Pseudo code

1. Transmit packets from source to destination.

2. For every node on the ongoing route do If (Critical Energy Level of a Node $\mathrm{N}==\mathrm{C}_{\mathrm{E}}$ )

Then

Node $\mathrm{N}$ will generate a Warning Message to the source. Here Node $\mathrm{N}$ is any node on the ongoing route.

3. If a Warning Message from any routing host is received, THEN

i. Source will not send a single packet in this current route.

ii. It will discard the current route from its cache.

iii. If any messages are need to be transmitted to destination then source will use another route from its cache.

Else

Source will forward packets on the current route.

This approach is shown graphically by considering the network in Figure 3. Where node $\mathrm{S}$ is a source, $\mathrm{D}$ is destination node and S using the primary route S-A-F-H-K$\mathrm{D}$ to send packets to $\mathrm{D}$ and each intermediate node simply relays the messages to latter on this route. This network shows energy of node $\mathrm{H}$ reduces to critical energy level.

In this case some messages are not reached to destination. Node A has message 6, node $\mathrm{F}$ has 4 and 5 and $\mathrm{H}$ have only message 3 which are shown in Figure $3 . \mathrm{H}$ generates a Warning Message $\mathrm{W}$ to $\mathrm{S}$ to tell that node $\mathrm{H}$ is an energy low node and to stop sending packets in this route by node $\mathrm{S}$ which is shown in Figure 4.

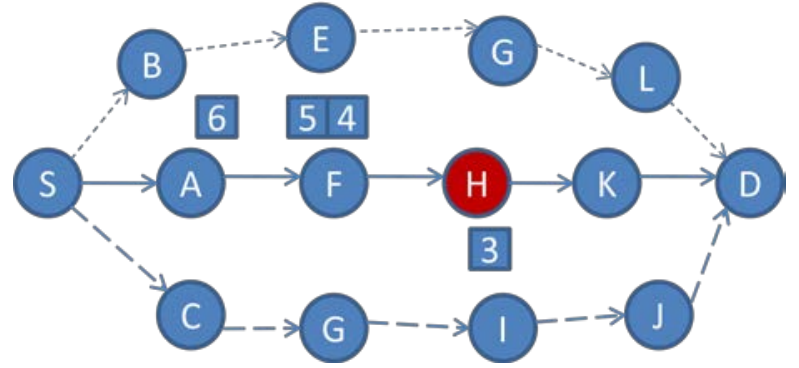

Fig. 3. Energy of Node H reduces to critical level
After receiving Warning Message $\mathrm{W}$, node $\mathrm{S}$ will not send a single packet using current route. By using the remaining energy node $\mathrm{H}$ tries its best to forward all the packets to the destination until its energy reduces to zero which is shown in Figure 5, Figure 6, Figure 7, and Figure 8. May be node $\mathrm{H}$ would not forward all the packets and it can be dead before forwarding all the packets to the destination. Thus there are some packets which may be lost. But node $\mathrm{H}$ will try its best to forward all the packets. Figure 9 shows that all the messages are forwarded by node $\mathrm{H}$ to node $\mathrm{K}$. At last energy of node $\mathrm{H}$ is reduced to zero and it is dead and vanished from the network and there is a broken route from $\mathrm{F}$ to $\mathrm{K}$ which is shown in Figure 10.

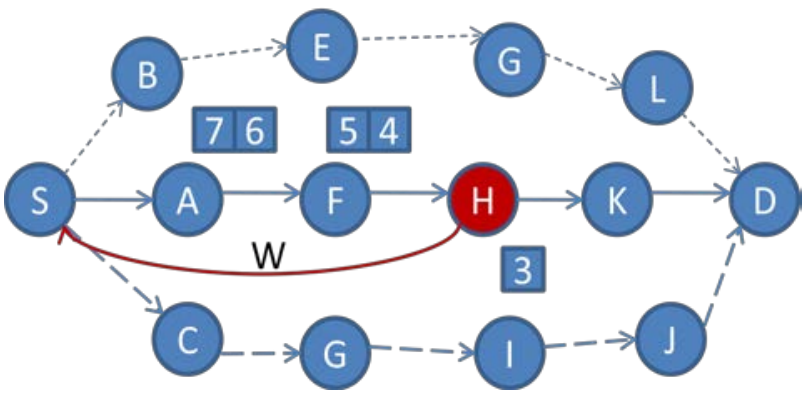

Fig. 4. $\mathrm{H}$ issues a Warning Message $\mathrm{W}$ to source $\mathrm{S}$

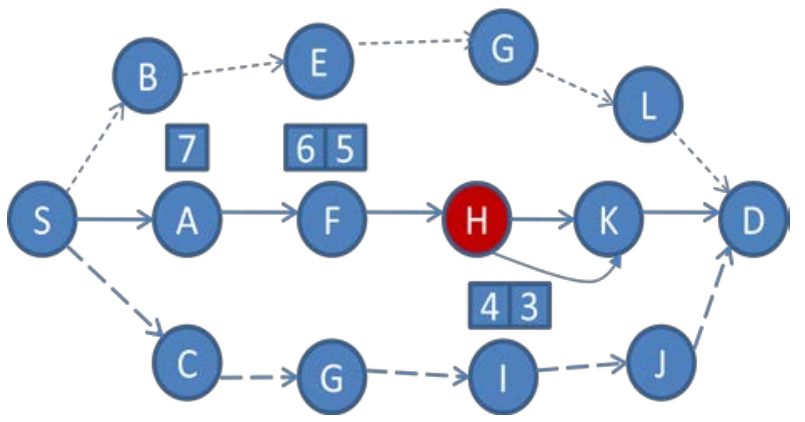

Fig. 5. $\mathrm{H}$ will forward message 3 to $\mathrm{K}$

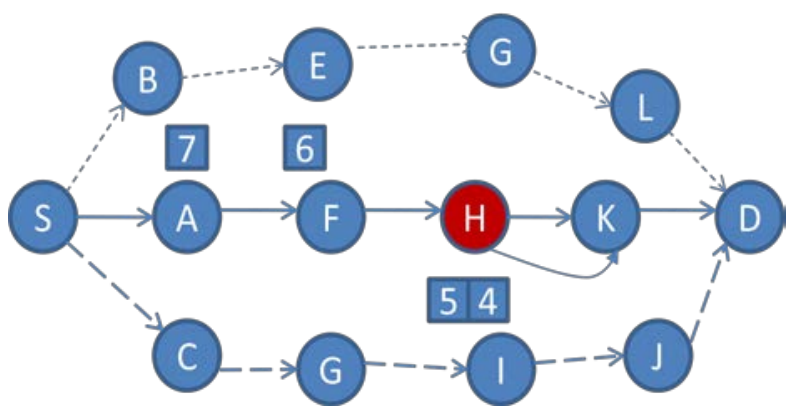

Fig. 6. $\mathrm{H}$ will forward the message 4 to $\mathrm{K}$

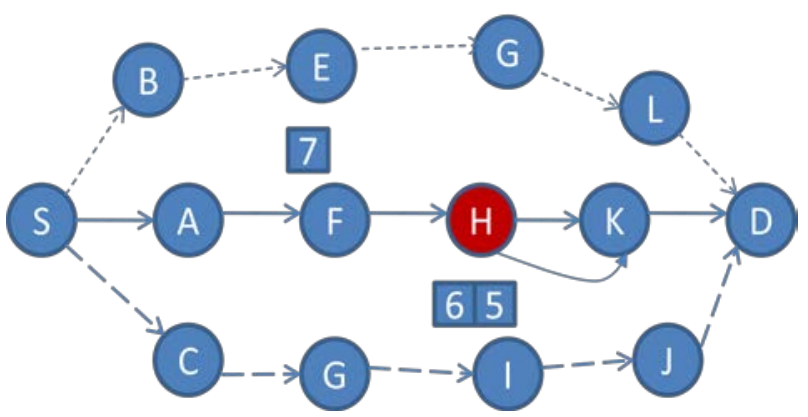

Fig. 7. $\mathrm{H}$ will forward the message 5 to $\mathrm{K}$ 


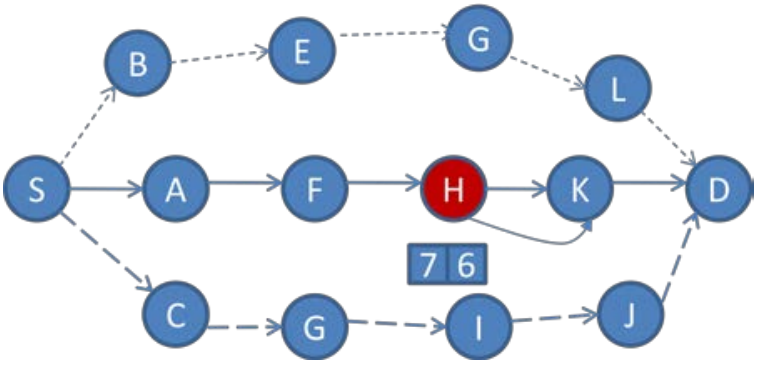

Fig. 8. $\mathrm{H}$ will forward the message 6 and 7 to $\mathrm{K}$

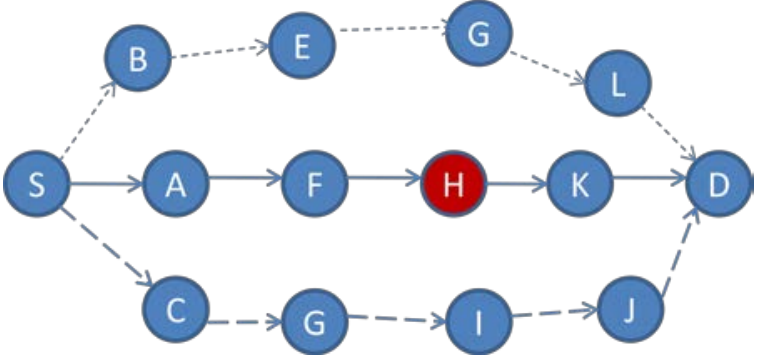

Fig. 9. $\mathrm{H}$ forwards all the messages to $\mathrm{K}$

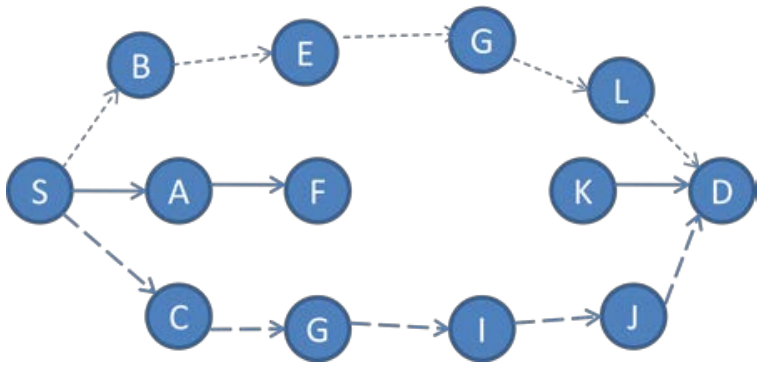

Fig. 10. Node $H$ is absent in the network

\section{Performance Comparison}

In dynamic source routing protocol ${ }^{3}$ and its extension ${ }^{4}$ when any route breakage event occurs ROUTE ERROR message is sent to the source. Until receiving any ROUTE ERROR message from any routing node source keeps continue sending packets on the current route. Upon receiving a ROUTE ERROR message source will stop sending packets on the ongoing route.

On the other hand proposed approach generates a Warning Message from a routing node whose energy level is at a point where only some messages can be forwarded by this node. Before finishing its energy to zero level it will generate this Warning Message advance to warn the source to stop sending packets on this route because this route will be broken soon. If source keeps continue sending packets on this route after receiving Warning Message these transmitted messages will be lost. The goal of generating Warning Message is to inform source to minimize packet losses in the network.

A scenario has been considered to compare these two approaches. In Figure 9 where no more messages are waiting in the intermediate nodes to be forwarded to D. But if $\mathrm{S}$ sends some more packets in this ongoing route there will be a route breakage event as it is done in $\mathrm{DSR}^{3}$ such as message 8 and 9 which is shown in Figure 11. These messages do not find a route to destination and these will be lost from the network because of a route error. In our approach source will never send these messages on the current route rather messages 8 and 9 are transmitted on different route S-B-E-G-L-D by the source which is shown in Figure 12. Finally these messages will reach to the destination if there is no problem occurs on this route. It is clear that if more messages like 10,11, and 12 are sent in their approach, these messages will be dropped at F. But in our approach these messages will never sent on this route.

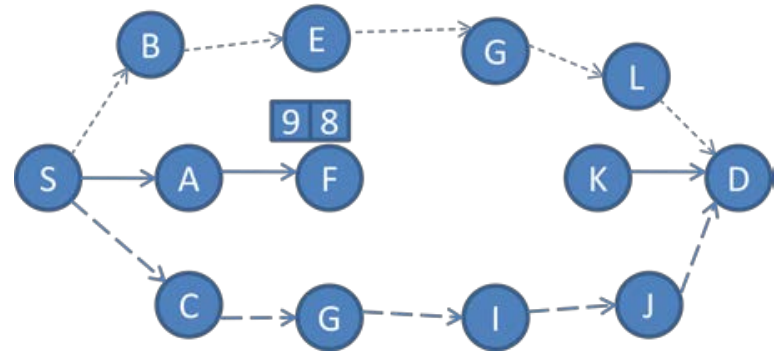

Fig. 11. Messages will be dropped in current approach

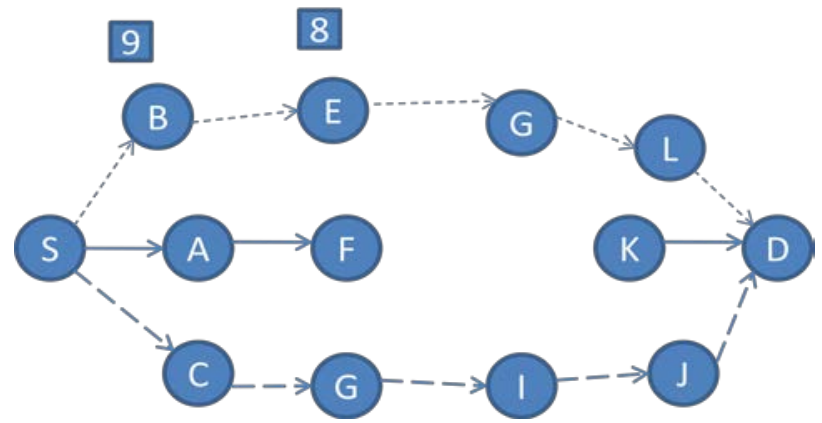

Fig. 12. Messages will reach to $D$ in our approach

A warning message is generated based on the critical energy level of a routing node.

If the critical energy level is set at a high value then unnecessarily routing node generates a Warning Message and if it is too low then there will be some messages which will never find a way to destination. So based on this assumption critical energy level is set $10 \%$ of node's total energy. Table 1 shows a contrast between $\mathrm{DSR}^{3}$ and its extension ${ }^{4}$ and DSR with our approach using the packets from network scenarios specified in Figure 4 to Figure 8 and Figure 11. From this Table 1 we can conclude in DSR more packets are lost than our approach.

Table 1. A comparison on $\operatorname{DSR}^{3}$ with its extension ${ }^{4}$ and $\mathrm{DSR}^{3}$ with proposed approach

\begin{tabular}{|l|l|l|}
\hline $\begin{array}{c}\text { Packets } \\
\text { No. }\end{array}$ & $\begin{array}{l}\text { DSR }^{3} \text { with its } \\
\text { extension }^{4}\end{array}$ & \multicolumn{1}{c|}{$\begin{array}{c}\mathrm{DSR}^{3} \text { with proposed } \\
\text { approach }\end{array}$} \\
\hline $\begin{array}{l}\text { 4, 5, 6, } \\
7\end{array}$ & forwarded & forwarded \\
\hline $\begin{array}{l}\text { 8, 9 or } \\
\text { more }\end{array}$ & $\begin{array}{l}\text { Dropped at } \\
\text { node F. }\end{array}$ & $\begin{array}{l}\text { Packets are sent using S-B- } \\
\text { E-G-L-D or some other route }\end{array}$ \\
\hline
\end{tabular}

\section{Conclusion}

This paper introduces two different concepts on mobile ad hoc network. One is the critical energy level of a routing node and another is the generating Warning Message by any routing node to the source to stop sending packets on the 
current route. When the source receives Warning Message it will stop sending packets on the current route. There will be less packet losses if our approach is incorporated with routing protocol such as dynamic source routing. Because source will stop sending packets on the current route whenever it receives Warning Message from any routing node. On the other hand in $\mathrm{DSR}^{3}$ source keeps transmitting packets on the current route until the arrival of ROUTE ERROR message. ROUTE ERROR message arrives later at the eleventh hour when there is no way to communicate with the current route but Warning Message comes earlier when routing node have $10 \%$ of its total energy. Thus less number of packets will be lost than DSR. The low energy node tries it best to forward all the messages of source until its death.

\section{References}

1. Hedrick, C., 1988. Routing Information Protocol. Internet Request For Comments 1058.
2. Moy, J., 1991. OSPF Version 2. Internet Request For Comments 1247.

3. Johnson, D. B., and D. A. Maltz, 1996. Dynamic source routing in ad hoc wireless networks. Kluwer International Series in Engineering and Computer Science, 153-179.

4. Wu, J., 2003. An extended dynamic source routing scheme in ad hoc wireless networks. Telecommunication Systems, 22(1-4), 61-75.

5. Perkins, C., Belding-Royer, E., and S. Das, 2003. Ad hoc on demand distance vector (AODV) routing (RFC 3561). IETF MANET Working Group.

6. Perkins, C. E., and P. Bhagwat, 1994. Highly dynamic destination-sequenced distance-vector routing (DSDV) for mobile computers. In ACM SIGCOMM Computer Communication Review 24(4), 234-244.

7. Haas, Z. J., Pearlman, M. R., and P. Samar, 2002. The zone routing protocol (ZRP) for ad hoc networks. Internet Draft. http://www.ietf.org/proceedings//55/1-D/draftieft-manet-zone-zrp-04.txt. 